\title{
ESTPiper - a web-based analysis pipeline for expressed sequence
} tags

\author{
Zuojian Tang, Jeong-Hyeon Choi, Chris Hemmerich, Ankita Sarangi, \\ John K Colbourne* and Qunfeng Dong*
}

Address: The Center for Genomics and Bioinformatics, Indiana University, Bloomington, Indiana, USA

Email: Zuojian Tang - tangz@cgb.indiana.edu; Jeong-Hyeon Choi - jeochoi@cgb.indiana.edu; Chris Hemmerich - chemmeri@cgb.indiana.edu; Ankita Sarangi - sarangia@indiana.edu; John K Colbourne* - jcolbour@cgb.indiana.edu; Qunfeng Dong* - qfdong@cgb.indiana.edu

* Corresponding authors

Published: 21 April 2009

BMC Genomics 2009, 10:174 doi:10.1186/147|-2164-10-174
Received: 26 September 2008

Accepted: 21 April 2009

This article is available from: http://www.biomedcentral.com/I47I-2/64/I0/I74

(c) 2009 Tang et al; licensee BioMed Central Ltd.

This is an Open Access article distributed under the terms of the Creative Commons Attribution License (http://creativecommons.org/licenses/by/2.0), which permits unrestricted use, distribution, and reproduction in any medium, provided the original work is properly cited.

\begin{abstract}
Background: EST sequencing projects are increasing in scale and scope as the genome sequencing technologies migrate from core sequencing centers to individual research laboratories. Effectively, generating EST data is no longer a bottleneck for investigators. However, processing large amounts of EST data remains a non-trivial challenge for many. Web-based EST analysis tools are proving to be the most convenient option for biologists when performing their analysis, so these tools must continuously improve on their utility to keep in step with the growing needs of research communities. We have developed a web-based EST analysis pipeline called ESTPiper, which streamlines typical large-scale EST analysis components.
\end{abstract}

Results: The intuitive web interface guides users through each step of base calling, data cleaning, assembly, genome alignment, annotation, analysis of gene ontology (GO), and microarray oligonucleotide probe design. Each step is modularized. Therefore, a user can execute them separately or together in batch mode. In addition, the user has control over the parameters used by the underlying programs. Extensive documentation of ESTPiper's functionality is embedded throughout the web site to facilitate understanding of the required input and interpretation of the computational results. The user can also download intermediate results and port files to separate programs for further analysis. In addition, our server provides a time-stamped description of the run history for reproducibility. The pipeline can also be installed locally, allowing researchers to modify ESTPiper to suit their own needs.

Conclusion: ESTPiper streamlines the typical process of EST analysis. The pipeline was initially designed in part to support the Daphnia pulex cDNA sequencing project. A web server hosting ESTPiper is provided at http://estpiper.cgb.indiana.edu/ to now support projects of all size. The software is also freely available from the authors for local installations.

\section{Background}

Expressed sequence tags (ESTs) are generated by singlepass sequencing of complementary DNA (cDNA) [1].
Because ESTs correspond to the transcribed regions of a genome, EST sequencing has been a common strategy for gene discovery - especially for organisms with complex 
genomes. For example, many agriculturally important plants (e.g., sunflower, wheat) have enormous genomes containing many repetitive elements and large intergenic regions. For these taxa, EST sequencing remains (for now) the only efficient way to discover genes on genome-wide scale, since the repetitive elements still pose an unsolved challenge for whole genome assembly. Even for species with draft genome sequences, ESTs remain the gold standard for accurate gene structure annotations (delineating intron-exon and gene boundaries) and serve a variety of biological research applications (reviewed in [2-4]). Because of improved reliable protocols for cDNA library construction, normalization and sequencing, generating EST data is now standard practice. As a result, the number of EST sequences is growing at an ever-increasing pace for diverse organisms [5]. After EST sequencing, the next step is to analyze the generated EST data. Typical EST analysis involves (i) base calling to convert raw chromatograms generated by DNA sequencers into human-readable sequences, (ii) data cleaning that removes cloning vector, adaptor and bacterial host sequence contamination, (iii) assembling individual EST sequences into contigs that reduce redundancy and represent a unique gene set, (iv) functional annotations of the potential encoded proteins by sequence similarity to annotated proteomes, and (v) designing microarray oligonucleotide probes from the EST sequences for expression profiling. For many of the above tasks, bioinformatics groups that specialize in EST data (e.g., PlantGDB [6]) usually implement their own inhouse EST analysis pipelines. However, those pipelines are generally not portable or accessible to outside users, due to specialized hardware requirement (such as parallel computers). Many biologists with smaller-scale EST projects can not afford dedicated bioinformatics teams or computational clusters. Therefore, individual biologists can face significant challenges to process and analyze their EST data. These include identifying, installing and executing the proper computer programs for each step. This is especially challenging if data processing requires additional programming (e.g., converting the output of one program to the required format for input into the next program). In most cases, significant computational resources are also needed (e.g., fast computers with enough memory and disk space). Therefore, web-based EST analysis pipelines are critical for biologists to perform their analysis simply, via a web browser, without unnecessary technical hassles. Although some web-based tools for EST analysis are becoming available, their scope and capacities need continuous improvements. To enrich the biologists' toolkit, we have developed a web-based computational pipeline called ESTPiper, which streamlines typical EST analysis steps. In the sections below, we discuss the technical implementation of ESTPiper, its unique features compared to other web-based EST analysis tools, and the application of ESTPiper in support of the Daphnia pulex genome sequencing project http://wFleaBase.org.

\section{Implementation}

The ESTPiper flow chart is illustrated in figure 1. The intuitive web interface guides the researcher through each step: base-calling, data cleaning, assembly, genome alignment, annotation, GO functional analysis, and microarray oligonucleotide probe design. At each step, the user sets the parameters to be used by the underlying analysis programs. Once the computation is completed, the user is notified via email and given a URL for viewing and downloading the results. For convenience, the results are temporarily stored on our server for 60 days. Each specific component of ESTPiper is described below.

\section{Base calling}

ESTPiper incorporates the Phred program [7] for base calling with quality scores, which is the de facto standard program for converting DNA sequence trace files (i.e., chromatograms) generated by the Sanger method into nucleotide sequences. To speed up the file upload for large numbers of trace files, ESTPiper only accepts compressed data files in either. $a b 1$ or .scf format and produces the sequence and the corresponding quality file in FASTA format.

\section{Data cleaning}

To obtain high quality EST assemblies, contaminant sequences are properly identified and removed. These include sequences from ligated adaptors, cloning vectors and the bacterial host. ESTPiper first invokes the commonly used LUCY program [8] for vector removal and to trim low quality regions at both ends of sequence reads. In addition, the user has the option to trim polyA/T tails from the sequence reads, which is a necessary step to avoid mis-assembly but optional if the user intends to identify transcriptional termination sites on the genome. Finally, the sequences are compared against bacterial genomes (downloaded from GenBank FTP site [9]) and other adapters and primers (UniVec [10]) based on stringent BLAST searches (i.e., E-value cutoff $1 \times 10^{-20}$ ). The output of this step is the cleaned sequence and quality files in FASTA format.

\section{Assembly}

ESTs usually correspond to only partial cDNA sequences and they are typically redundant, even when normalized during library construction. Therefore, overlapping EST sequences are commonly assembled to derive a set of unique putative genes (unigenes). ESTPiper provides de novo assembly using the popular CAP3 program [11] to assemble ESTs into contigs based on mutual percent identity over a minimum number of overlapping bases. In addition, if the 5' and 3' ESTs derived from the same 


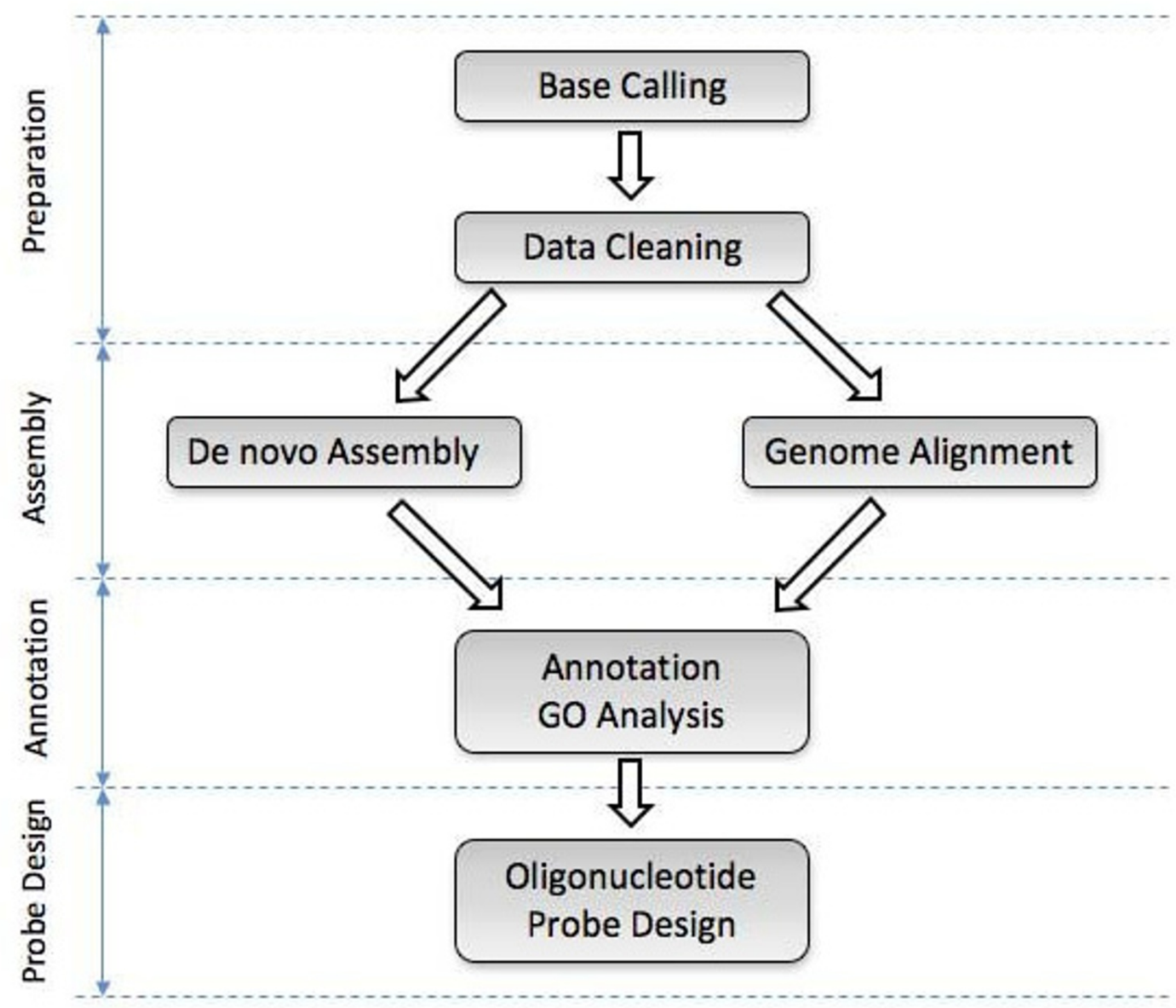

Figure I

Schematic overview of ESTPiper. See text for detailed description.

cDNA clones follow the standard naming convention (e.g., .fwd and .rev for sequences generated from forward and reverse sequencing primers, respectively), such clonepair information can be used to produce unigene clusters that include non-overlapping contigs. For this purpose, ESTPiper performs single-linkage clustering by default. The user may choose a more stringent clustering criterion, i.e., at least two EST clones must be shared for linking two contigs together in order to reduce potential false linkages (similar to other practice, e.g., [12]).

\section{Genome alignment}

If a draft genome sequence for the species of interest is available, ESTs are routinely aligned to genomic DNA for gene discovery, for annotation of intron-exon structures, and for identifying alternative splice forms. For such purposes, we have also implemented a genome-alignment module. Specifically, the BLAT program [13], which is designed to align native or closely related ESTs to the genome, is called by ESTPiper to perform spliced alignment of each EST against user-supplied genome sequences. If an EST sequence matches multiple genomic loci, only the best match is considered as the cognate match. After ESTs are aligned to genomic DNA, the mapping coordinates (i.e., the start and end position of each aligned EST on genomic scaffolds) allow the user to cluster overlapping ESTs into unigene sets. Genome-based EST clustering is usually considered more accurate than de 
novo assembly [12,14-16]. Without draft genome sequences for guidance, de novo assemblers solely consider the pairwise EST sequence overlap, and may mistakenly assemble different transcripts from paralogous genes into the same cluster, which is a serious problem for species that have extensive gene duplicates. Moreover, de novo assemblers often disconnect alternative transcripts derived from the same gene locus into different clusters, thus overestimating the number of expressed genes. Therefore, we have implemented a genome-guided strategy for clustering the mapped ESTs similar to other published studies (e.g., $[12,16])$. Particularly, ESTs aligned to the same genomic locus are clustered based on user-supplied parameters, e.g., minimum number of overlapping nucleotides between the neighboring aligned EST sequences. Then, transcript sequences can be derived based on the exons defined by each matched EST produced by the BLAT program. Specifically, ESTPiper invokes the well-known PASA package to generate contigs for each alternative splice variant by merging sets of compatible overlapping EST alignments [17]. If multiple splice variants are identified, all will be reported. However, only the longest splice variant from each gene cluster is selected as the representative of the gene transcript for the following modules (i.e., functional annotation and microarray oligonucleotide probe design).

\section{Functional Annotation}

The next natural step when processing ESTs is to identify the potential protein products encoded by the clustered unigene sequences, particularly whether they are similar to known sequences in proteomic databases. Therefore, each assembled contig sequence is searched against the UniProt protein database [18] using the BLAST program [19] in ESTPiper. A local copy of the UniProt database is automatically synchronized with the UniProt server via a monthly cron job. The UniProt database has the advantage over other comprehensive datasets for annotation of providing gene ontology (GO) terms in its protein records [20]. GO terms associated with the top statistically significant database sequence matches are propagated in ESTPiper to the contig sequence following common practice (e.g., [21]). The GO terms allow biologists to conveniently summarize the gene product attributes of their sequences with controlled vocabulary. ESTPiper allows the user to create a summary of GO terms in tabular format based on different GO categories (i.e., biological process, molecular function, and cellular component) and at a user-selected GO hierarchical level. ESTPiper also allows the user to input a list of GO terms (e.g., GO terms derived from genes unique to a particular EST library) and reference set (e.g., GO terms corresponding to the entire transcriptome), and to perform statistical analyses that identify overrepresented functional attribute terms. Specifically, the p-value of each GO term is calculated based on the hypergeometric test with Benjamini and Hochberg multiple testing correction [22].

\section{Microarray oligonucleotide probe design}

Despite increasing popularity of whole-genome tiling arrays and expression profiling by direct sequencing, oligonucleotide arrays remain an efficient and cost effective tool for studying co-transcriptional biases of genes on a large scale for species without full-genome sequence information. Therefore, designing microarray oligonucleotide probes is a critical task for biologists to create gene chips for their functional genomic investigations. Previously, our Center conducted an extensive survey to compare the existing microarray probe design programs [23] and chose the OligoPicker program [24] to successfully design microarray probes within ESTPiper for a number of expression profiling studies in different species (e.g., Coprinus cinereus, Daphnia pulex). The coding strand (i.e., sense strand) of each assembled sequence must be predefined before designing microarray probes, which is seldom straightforward. In ESTPiper, the coding strand is determined by a simple two-tier strategy. First, sequences on DNA strands that match to the protein database (e.g., through the above Functional Annotation module), are confidently determined from the BLAST output. If a sequence has no match to protein sequences (e.g., EST sequences representing novel species-specific genes), the sequence is passed to the OrfPredictor program [25], which identifies the longest open reading frame to predict the coding strand.

\section{Automated Pipeline}

Each of the above steps is completely modularized. Therefore, depending on the user's specific needs, multiple entry points into the pipeline are possible. For example, instead of starting with base calling for processing trace files, the user can simply upload pre-processed FASTA-format sequence files and quality scores (perhaps generated by others) into our pipeline for assembly. Similarly, intermediate results can be downloaded from our pipeline (e.g., assembled contig sequences) to be imported within other preferred computer programs for further analysis (e.g., functional annotation). In addition, ESTPiper provides an option for the user to select and combine multiple (or all) modules automatically. Data can be effectively transferred among adjacent modules without any human intervention. Parameters can be saved for repeated use, i.e., the user can input previously returned parameter files for multiple new runs with different source data.

\section{Results and discussion Comparison with other existing EST analysis programs}

A number of standalone software packages are available for EST data analysis [26-37]. However, those programs require researchers to install and maintain the software 
locally, which many biologists find inconvenient (e.g., certain prerequisites can present a serious challenge to install and update, even for bioinformaticians). Instead, biologists often prefer web-based analysis tools, where data can be uploaded on a host machine and the analysis can be performed through an easy-to-use interface. Therefore, several online tools have been published recently that simplify computational tasks. Although helpful, none of the existing web-based tools offer a comprehensive EST analysis workflow. For example, many tools are limited in scope: e.g., OREST [38] is only designed for processing mammalian and fungal sequences, not necessarily applicable to other research communities. In addition, OREST does not provide some of the critical modules in ESTPiper (e.g., de novo EST assembly, microarray probe design). For general-purpose EST analysis tools, preAssemble [39] and WebTraceMiner [40] specialize in base calling and quality trimming but do not provide assembly or annotation functionality. EGassembler [41] mainly focuses on de novo EST assembly but users must perform base calling and annotation elsewhere. ESTExplorer [42] and ESTpass [43] extend EGassember with additional functional annotations, but these programs lack genome alignment and microarray probe design functionality. Here, we present an alternative EST analysis pipeline, ESTPiper, for research communities. In addition to streamlining the steps of base calling, quality trimming and removing contaminant sequences, assembly, annotation and GO analysis, ESTpiper provides two unique modules compared to existing web-based EST analysis tools: (i) genome alignment and (ii) microarray probe design. As discussed above, both functions are now standard practice in a typical EST project. However, these functions are not available in existing EST analysis tools (Table 1). Although standalone web servers exist for similar tasks (e.g., e2g [44] for genome alignment and PROBEmer [45] for probe design), ESTPiper integrates such functionalities into a comprehensive pipeline with additional enhancements. For the genome alignment module, the existing tools for EST to genome alignment do not provide customizable clustering function. ESTPiper allows users to specify how ESTs aligned to the genome should be clustered, by defining a minimum distance between the neighboring aligned EST sequences. Moreover, such clus- tering can be improved by providing clone pair information. For the probe design component, all existing probe design software requires researchers to identify the coding strand before executing. However, for a large number of EST-derived sequences, it is not trivial for biologists to determine the correct strand for each. Therefore, we integrated the database similarity search (i.e., BLAST) and $a b$ initio prediction (i.e., OrfPredictor) for coding strand determination. In addition, we also enhanced the de novo assembly function. Specifically, beyond simply invoking CAP3 as other services do, ESTpiper provides users an option to perform single-linkage clustering based on clone-pair constraints. This allows the user to better define a true set of unigenes (i.e., ESTs derived from the same cDNA clones, even if the sequences do not overlap). Furthermore, some of existing web-based tools allow researchers to process only relatively small input files. For example, ESTpass imposes an upper limit of 10,000 ESTs (or 20 Mbyte file size) on files uploaded to their web server. We impose no file size limit for ESTPiper. However, we do recommend that users input less than 100,000 EST sequences to ensure successful de novo assembly without running out of computer memory on our current server. Yet we have nonetheless successfully assembled more than 150,000 Daphnia ESTs with our present configuration.

\section{Other features}

Our workbench is designed for biologists to perform and document computational analysis on EST data. Computational analyses in ESTPiper are documented for reproducibility. For example, the percent identity cutoff limits used by CAP3 program for assembly are recorded, which determines the resulting contigs. Therefore, ESTPiper provides the user with a complete, time-stamped description of ESTPiper's usage history (e.g., the programs, parameters, input data, and corresponding results). We believe that this feature will greatly facilitate tracking results, especially if the user initiates several rounds of trial-and-error analyses, experimenting with different program and parameter combinations in order to obtain the highest quality results. Finally, unlike many other web-based tools that are not portable, the user can download and install ESTPiper on local computers. For example,

Table I: Comparison of the available features of ESTPiper with other web-based EST analysis tools.

\begin{tabular}{lllllll}
\hline Web-based EST analysis pipeline & preAssemble & EGassembler & ESTExplorer & ESTpass & WebTraceMiner & ESTPiper \\
\hline Base calling & Yes & No & No & No & Yes & Yes \\
Data cleaning & Yes & Yes & Yes & Yes & Yes \\
De novo assembly & No & Yes & Yes & Yes & No & Yes \\
Genome alignment & No & No & No & No & No & Yes \\
Annotation & No & No & Yes & Yes & No & Yes \\
GO analysis & No & No & Yes & Yes & No & No \\
Probe design & No & No & No & No & Yes
\end{tabular}


advanced users may use the core ESTPiper code to process the EST data without having to navigate through the web interface, or they can integrate ESTPiper into their own customized pipeline (e.g., replace any individual modules in ESTPiper with their preferred analysis programs).

\section{Application}

We applied ESTPiper to process and analyze a large set of Daphnia pulex EST data as part of the Daphnia Genomics Consortium sequencing project. We began our data analysis with 151,111 EST sequences that were filtered from an initial set of 219,948 trace files that were generated by sequencing 37 cDNA libraries for discovering condition specific gene transcripts (detailed analysis of these results is presented elsewhere). After data cleaning, ESTPiper returned 151,013 high-quality sequences. PolyA/T tails were further removed; the minimum length of continuous polyA/T was set to $9 \mathrm{bp}$, the maximum number of mismatches within the polyA/T region was 3, the searching range of polyA/T was limited to $50 \mathrm{bp}$ from both ends of the sequence. We also configured ESTPiper to remove sequences with at least $30 \mathrm{bp}$ continuous $\mathrm{A} / \mathrm{T}$ or adaptors occurring in the middle of sequence reads to avoid potential chimerical clones. Furthermore, mitochondrial sequences and contaminated E. coli sequences were identified and removed based on BLAST similarity search (Evalue cutoff $\left.1 \times 10^{-10}\right)$. Finally, resulting sequences less than 100 bp were also removed. A total of 148,410 highquality ESTs were therefore used in subsequent steps of our analysis.

We conducted both a de novo assembly using the CAP3 program and an assembly based on alignment to the Dappu v1.1 draft genome sequence assembly (September, 2006). First, by feeding the cleaned ESTs into the de novo CAP3 assembly program (with the parameters - $p 95$-o 49 -t 10000), 23,470 contigs and 14,014 singletons were generated, and 26,265 unigene clusters were derived based on clone-pair constraint. Second, for genome-based assembly, ESTs were first aligned to the Daphnia genome using the BLAT program (with the parameter minIdentity $=95$ ). If an EST sequence matched multiple genomic loci, only the best match was considered as the cognitive match. Out of 148,410 ESTs, 113,931 ESTs matched to the genome sequence. ESTs were clustered based on their overlapping matching positions on the genome. We required that two neighboring ESTs be considered part of the same cluster if they shared at least $40 \mathrm{bp}$ minimum overlap. A total of 14,891 unigene sets were derived. For genes identified from each EST library, ESTPiper matched them to UniProt using BLASTX (E-value cutoff is $1 \times 10^{-20}$ ). The GO term associated with the top matches to the protein database were also created for different libraries. Statistic analysis of GO terms overrepresented in each library was performed using the entire EST collection as a reference. Finally, a
10,000 element Daphnia cDNA microarray (Generation3) was produced with the oligonucleotide probes designed based on ESTPiper. The microarray has been successfully applied by the Daphnia research community to study Daphnia gene expression under different environmental stress conditions (data will be published elsewhere).

\section{Conclusion}

Web-based tools are most convenient for biologists to effectively process large EST data sets. To supplement the existing tools, we have developed a comprehensive webbased EST analysis pipeline called ESTPiper that streamlines the numerous EST analysis components and offers unique features such as genome alignment and microarray probe design.

\section{Availability and requirements}

The ESTPiper program is freely accessible, using a web browser at http://estpiper.cgb.indiana.edu/. We recommend that users provide their email address when they upload their data. Then, once their submitted jobs are finished, emails will be automatically sent to the users with the instruction for retrieving their results. The software is also available from the web site for local installation. Currently, ESTPiper is installed on a virtual machine hosted on a Sun X4450 with four 2.4 GHz CPUs, each CPU having four cores for 16 total cores. The machine has $32 \mathrm{~GB}$ of memory. There are two $10 \mathrm{~K}$ RPM SAS system disks in a mirrored ZFS pool, and all project/app storage is done over NFS via dedicated gigabit Ethernet. At our Center, we can easily migrate ESTPiper among our virtual servers as resource requirements change.

\section{Project name: ESTPiper}

\section{Project home page: http://estpiper.cgb.indiana.edu/}

Operating systems: Local installation requires Linux/ UNIX.

Programming language: Perl, JavaScript, JAVA

License: The software is under the Apache license 2.0.

\section{Authors' contributions}

ZT designed and implemented ESTPiper and its web server. JHC contributed to the system design and provided technical assistance with the software implementation. $\mathrm{CH}$ critically enhanced the web interface, on-line descriptions, and the probe design module. AS and $\mathrm{CH}$ improved the genome alignment module. QD and JC conceived the project and guided the development process. QD and ZT prepared the manuscript. All authors read and approved the final manuscript. 


\section{Acknowledgements}

This work was supported in part by the Indiana METACyt Initiative of Indiana University, funded in part through a major grant from the Lilly Endowment, Inc. The project was also supported by the National Science Foundation grant 0221837 "Development of Methods Linking Genomic and Ecological Responses in a Freshwater Sentinel Species", and by the Indiana Center for Insect Genomics project funded through the Indiana 2 Ist Century Research and Technology Fund. We thank Amanda Hemmerich for improving ESTPiper web interface, Rupali Patwardhan for her work on microarray probe design and Phillip Steinbachs for system administrative support. The sequencing was performed at the DOE Joint Genome Institute under the auspices of the U.S. Department of Energy's Office of Science, Biological and Environmental Research Program, and by the University of California, Lawrence Livermore National Laboratory under Contract No. W-7405-Eng-48, Lawrence Berkeley National Laboratory under Contract No. DE-AC02-05CHI I23I, Los Alamos National Laboratory under Contract No. W-7405-ENG-36 and in collaboration with the Daphnia Genomics Consortium (DGC) http://daphnia.cgb.indiana.edu. Our work benefits from, and contributes to the Daphnia Genomics Consortium.

\section{References}

I. Adams MD, Kelley JM, Gocayne JD, Dubnick M, Polymeropoulos MH, Xiao H, Merril CR, Wu A, Olde B, Moreno RF, et al:: Complementary DNA sequencing: expressed sequence tags and human genome project. Science |99|, 252(50 13): $165 \mid-1656$.

2. Rudd S: Expressed sequence tags: alternative or complement to whole genome sequences? Trends Plant Sci 2003, 8(7):321-329.

3. Dong Q, Kroiss L, Oakley FD, Wang BB, Brendel V: Comparative EST analyses in plant systems. Methods Enzymol 2005, 395:400-418.

4. Nagaraj SH, Gasser RB, Ranganathan S: A hitchhiker's guide to expressed sequence tag (EST) analysis. Brief Bioinform 2007, 8(I):6-2I

5. dbEST: database of Expressed Sequence Tags [http:// www.ncbi.nlm.nih.gov/dbEST/dbEST summary.html]

6. Dong Q, Lawrence CJ, Schlueter SD, Wilkerson MD, Kurtz S, Lushbough C, Brendel V: Comparative plant genomics resources at PlantGDB. Plant Physiol 2005, 139(2):610-618.

7. Ewing B, Hillier L, Wend I MC, Green P: Base-calling of automated sequencer traces using phred. I. Accuracy assessment. Genome Res 1998, 8(3): 175-185.

8. Chou $\mathrm{HH}$, Holmes $\mathrm{MH}$ : DNA sequence quality trimming and vector removal. Bioinformatics 200 I, I 7( I 2): I093-I I04.

9. NCBI FTP Site [http://www.ncbi.nlm.nih.gov/Ftp/]

10. The UniVec DataBase [http://www.ncbi.nlm.nih.gov/VecScreen/ UniVec.html]

II. Huang X, Madan A: CAP3: A DNA sequence assembly program. Genome Res 1999, 9(9):868-877.

12. Kim N, Shin S, Lee S: ECgene: genome-based EST clustering and gene modeling for alternative splicing. Genome Res 2005, I 5(4):566-576.

13. Kent W]: BLAT-the BLAST-like alignment tool. Genome Res 2002, I 2(4):656-664.

14. Zhu W, Schlueter SD, Brendel V: Refined annotation of the Arabidopsis genome by complete expressed sequence tag mapping. Plant Physiol 2003, I32(2):469-484.

I5. Brendel V, Xing L, Zhu W: Gene structure prediction from consensus spliced alignment of multiple ESTs matching the same genomic locus. Bioinformatics 2004, 20(7): I I57-I I69.

16. Jain M, Shrager J, Harris EH, Halbrook R, Grossman AR, Hauser C, Vallon O: EST assembly supported by a draft genome sequence: an analysis of the Chlamydomonas reinhardtii transcriptome. Nucleic Acids Res 2007, 35(6):2074-2083.

17. Haas BJ, Delcher AL, Mount SM, Wortman JR, Smith RK Jr, Hannick LI, Maiti R, Ronning CM, Rusch DB, Town CD, et al.: Improving the Arabidopsis genome annotation using maximal transcript alignment assemblies. Nucleic Acids Res 2003, 3 I ( I 9):5654-5666.

18. The universal protein resource (UniProt). Nucleic Acids Res 2008:DI90-195.
19. Altschul SF, Madden TL, Schaffer AA, Zhang J, Zhang Z, Miller W, Lipman DJ: Gapped BLAST and PSI-BLAST: a new generation of protein database search programs. Nucleic Acids Res 1997, 25( I 7):3389-3402.

20. The Gene Ontology project in 2008. Nucleic Acids Res 2008:D440-444.

21. Gotz S, Garcia-Gomez JM, Terol J, Williams TD, Nagaraj SH, Nueda MJ, Robles M, Talon M, Dopazo J, Conesa A: High-throughput functional annotation and data mining with the Blast2GO suite. Nucleic Acids Res 2008, 36( I 0):3420-3435.

22. Benjamini $Y$, Hochberg $Y$ : Controlling the false discovery rate-a practical and powerful approach to multiple testing. J $R$ Stat Soc Ser 1995, 57:289-300.

23. Oligo Design - Survey Of Available Programs [https:// projects.cgb.indiana.edu/display/brp/Oligo+Design+Survey]

24. Wang X, Seed B: Selection of oligonucleotide probes for protein coding sequences. Bioinformatics 2003, I9(7):796-802.

25. Min XJ, Butler G, Storms R, Tsang A: OrfPredictor: predicting protein-coding regions in EST-derived sequences. Nucleic Acids Res 2005:W677-680.

26. Ayoubi P, Jin X, Leite S, Liu X, Martajaja J, Abduraham A, Wan Q, Yan W, Misawa E, Prade RA: PipeOnline 2.0: automated EST processing and functional data sorting. Nucleic Acids Res 2002, 30(2I):476I-4769.

27. Mao C, Cushman JC, May GD, Weller JW: ESTAP-an automated system for the analysis of EST data. Bioinformatics 2003, I9(13): I720-1722.

28. Paquola AC, Nishyiama MY Jr, Reis EM, da Silva AM, Verjovski-Almeida S: ESTWeb: bioinformatics services for EST sequencing projects. Bioinformatics 2003, I 9( I 2): I 587-I 588.

29. Scheetz TE, Trivedi N, Roberts CA, Kucaba T, Berger B, Robinson NL, Birkett CL, Gavin AJ, O'Leary B, Braun TA, et al.: ESTprep: preprocessing cDNA sequence reads. Bioinformatics 2003, I9(I I): $1318-1324$.

30. Hotz-Wagenblatt A, Hankeln T, Ernst P, Glatting KH, Schmidt ER, Suhai S: ESTAnnotator: A tool for high throughput EST annotation. Nucleic Acids Res 2003, 3 I ( I3):3716-37I9.

31. Pertea G, Huang X, Liang F, Antonescu V, Sultana R, Karamycheva S, Lee Y, White J, Cheung F, Parvizi B, et al.: TIGR Gene Indices clustering tools (TGICL): a software system for fast clustering of large EST datasets. Bioinformatics 2003, I 9(5):65 I-652.

32. Xu H, He L, Zhu Y, Huang W, Fang L, Tao L, Cai L, Zhang L, Zhou Y: EST pipeline system: detailed and automated EST data processing and mining. Genomics Proteomics Bioinformatics 2003, I(3):236-242

33. Parkinson J, Anthony A, Wasmuth J, Schmid R, Hedley A, Blaxter M: PartiGene-constructing partial genomes. Bioinformatics 2004, 20(9): | 398-|404.

34. Matukumalli LK, Grefenstette JJ, Sonstegard TS, Van Tassell CP: ESTPAGE-managing and analyzing EST data. Bioinformatics 2004, 20(2):286-288.

35. D'Agostino N, Aversano M, Chiusano ML: ParPEST: a pipeline for EST data analysis based on parallel computing. BMC Bioinformatics 2005, 6(Suppl 4):S9.

36. Zhu T, Zhou J, An Y, Li H, Xu G, Ma D: Construction and characterization of a rock-cluster-based EST analysis pipeline. Comput Biol Chem 2006, 30(I):8I-86.

37. Forment J, Gilabert F, Robles A, Conejero V, Nuez F, Blanca JM: EST2uni: an open, parallel tool for automated EST analysis and database creation, with a data mining web interface and microarray expression data integration. BMC Bioinformatics 2008, 9:5.

38. Waegele B, Schmidt T, Mewes HW, Ruepp A: OREST: the online resource for EST analysis. Nucleic Acids Res 2008:W I 40-I44.

39. Adzhubei AA, Laerdahl JK, Vlasova AV: preAssemble: a tool for automatic sequencer trace data processing. BMC Bioinformatics 2006, 7:22.

40. Liang C, Wang G, Liu L, Ji G, Liu Y, Chen J, Webb JS, Reese G, Dean JF: WebTraceMiner: a web service for processing and mining EST sequence trace files. Nucleic Acids Res 2007:WI37-I42.

41. Masoudi-Nejad A, Tonomura K, Kawashima S, Moriya Y, Suzuki M, Itoh M, Kanehisa M, Endo T, Goto S: EGassembler: online bioinformatics service for large-scale processing, clustering and assembling ESTs and genomic DNA fragments. Nucleic Acids Res 2006:W459-462. 
42. Nagaraj SH, Deshpande N, Gasser RB, Ranganathan S: ESTExplorer: an expressed sequence tag (EST) assembly and annotation platform. Nucleic Acids Res 2007:W 143-147.

43. Lee B, Hong T, Byun SJ, Woo T, Choi YJ: ESTpass: a web-based server for processing and annotating expressed sequence tag (EST) sequences. Nucleic Acids Res 2007:WI59-162.

44. Kruger J, Sczyrba A, Kurtz S, Giegerich R: e2g: an interactive webbased server for efficiently mapping large EST and CDNA sets to genomic sequences. Nucleic Acids Res 2004:W30I-304.

45. Emrich SJ, Lowe M, Delcher AL: PROBEmer: A web-based software tool for selecting optimal DNA oligos. Nucleic Acids Res 2003, 3 I (I3):3746-3750.

Publish with Bio Med Central and every scientist can read your work free of charge

"BioMed Central will be the most significant development for disseminating the results of biomedical research in our lifetime. "

Sir Paul Nurse, Cancer Research UK

Your research papers will be:

- available free of charge to the entire biomedical community

- peer reviewed and published immediately upon acceptance

- cited in PubMed and archived on PubMed Central

- yours - you keep the copyright 